\title{
Linx
}

Revue des linguistes de l'université Paris X Nanterre

66-67| 2012

Dynamiques de la construction des sens attendus et inattendus dans les langues

\section{Prises de distance en finnois : le mode jussif et le marqueur discursif $-h A n$}

\section{Outi Duvallon et Rea Peltola}

\author{
(2) OpenEdition \\ Journals \\ Édition électronique \\ URL : http://journals.openedition.org/linx/1453 \\ DOI : $10.4000 / \operatorname{linx} .1453$ \\ ISSN : 2118-9692 \\ Éditeur \\ Presses universitaires de Paris Nanterre \\ Édition imprimée \\ Date de publication : 15 septembre 2012 \\ Pagination : 101-116 \\ ISSN : 0246-8743 \\ Référence électronique \\ Outi Duvallon et Rea Peltola, «Prises de distance en finnois : le mode jussif et le marqueur discursif - \\ hAn », Linx [En ligne], 66-67 | 2012, mis en ligne le 15 septembre 2013, consulté le 19 avril 2019. URL \\ http://journals.openedition.org/linx/1453; DOI : 10.4000/linx.1453
}




\title{
Prises de distance en finnois : le mode jussif et le marqueur discursif $-h A n^{1}$
}

\author{
Outi Duvallon \\ INALCO/USPC, CNRS UMR8202 IRD UMR135, SeDyL \\ Rea Peltola \\ Normandie Université - UNICAEN, CRISCO (EA 4255)
}

\section{Introduction}

Cet article s’intéresse à la question du sens attendu/inattendu du point de vue des énoncés à valeur concessive. En étudiant deux formes finnoises, d'une part, le mode jussif en - $k O O n$ et, d'autre part, le marqueur discursif - $h A n$, nous développerons l'idée d'un procédé de distanciation permettant au locuteur de prendre en considération un état de choses sans y adhérer complètement.

Le mode jussif et le marqueur discursif $-h A n$ appartiennent à des catégories linguistiques différentes, le premier ayant trait à l'actualisation du procès (Guillaume, 1929), le deuxième, qui est d'origine pronominale (Laitinen, 2002), à la détermination de l'origo énonciative. Ces deux formes se rencontrent dans des contextes similaires, mais se combinent difficilement à l'intérieur d'un énoncé.

\footnotetext{
${ }^{1}$ Ce travail est en relation avec l'opération GD3 A cross-linguistic approach of discourse markers de l'axe 3 du Labex EFL (financé par ANR/CGI).
} 
Dans les exemples (1a) avec un énoncé jussif et (1b) avec un énoncé en - $h A n$, le locuteur se positionne par rapport à une intention qui n'est pas la sienne pour indiquer qu'il ne s'y oppose pas alors qu'on aurait pu s'attendre au contraire :

Exemple 1a :

1 He ovat nuoria ja kauniita. Minä olen vanha ja ruma. [lls sont jeunes et beaux, moi je suis vieux et laid.]

2 Mitäpä hän minusta, kun on semmoinen, joka ottaa. quand être-3 un (comme ça) REL prendre-3 [Pourquoi se soucierait-elle de moi quand c'est un homme comme ça qui la prend.] »

3 Otta-koon! Otta-koon! Pitä-köön! prendre-Jus prendre-Jus garder-Jus [Qu'il la prenne, qu'il la prenne et qu'il la garde.]

(Aho, 1911, VII) $)^{2}$

Exemple 1b :

1 Ei isänsä ensimmäisenä kolmena vuotena kehenkään muuhun koskenut. [Pendant les trois premières années, son père n'a regardé personne d'autre que moi.]

2 Olin nuori ja kaunis, olin kuudentoista, kun keikautti rekeensä. [J'étais jeune et belle, j'avais seize ans quand il me jeta dans son traîneau.]

3 Sitten vasta otti kesätyttöjä, là seulement prendre-PRET-3 été-fille-PL-PAR

4 kun minä lapsia imetin, quand je enfant-PL-PAR allaiter-PRET-1

[Ce fut seulement lorsque j'allaitais les enfants qu'il prit des filles pour l'été,]

5 ja sai-han ottaa, (...)

et pouvoir-PRET-3-hAn prendre

[et il powvait bien en prendre, $(\ldots)$ ]

(Aho, 1911, XI)

\footnotetext{
${ }^{2}$ La plupart des exemples cités dans cet article sont tirés d'un roman intitulé Juha (1911) de Juhani Aho (1861-1921), auteur originaire de l'Est de la Finlande et dont le texte reflète des caractéristiques des dialectes orientaux du finnois. En effet, aussi bien la forme jussive que le marqueur discursif $-h A n$ ont une présence plus importante dans les dialectes orientaux que dans les dialectes occidentaux et le finnois standard (Forsberg, à paraitre ; Hakulinen, 1999 (1951), p. 46 ; Forsman Svensson, 2003, p. 52). Le corpus donnant accès à une forme non standardisée de la langue nous a permis de mieux évaluer le potentiel sémantique des formes analysées que ne l'aurait fait un corpus de finnois standardisé.
} 
La forme jussive et le marqueur discursif $-h A n$ ont pour point commun de mettre en place une situation d'énonciation où le locuteur n'assume pas toute la responsabilité quant à l'état de choses exprimé. En effet, la prise en charge énonciative peut porter sur différents aspects de l'énoncé, notamment la mise en mots d'un état de choses, c'est-à-dire la construction d'une relation prédicative, et la validation modale de cette prédication (Dendale et Coltier, 2011, p. 8). Aussi bien le jussif que le marqueur discursif - $h A n$ impliquent que la responsabilité énonciative est partagée entre le locuteur et une troisième instance. De cela, il résulte une distance entre le locuteur et son dire.

Dans ce qui suit, nous examinerons d'abord les propriétés sémantiques de la forme jussive et du marqueur discursif $-h A n$. On verra que la position de la troisième instance, qui constitue un élément essentiel dans le procédé de distanciation, n'est pas la même dans les deux cas. Dans un deuxième temps sont étudiés deux types de figures discursives auxquelles les deux formes peuvent participer, soit comme des procédés concurrents, soit comme des procédés complémentaires qui se succèdent dans le texte. On tentera de déterminer, pour chacune de ces formes, la façon dont elle répond aux attentes et sa capacité de réorienter le discours.

\section{Présentation des formes}

\subsection{Le jussif}

La forme jussive qui est marquée par le suffixe -kOOn est considérée, dans les grammaires finnoises, comme la troisième personne de l'impératif (voir par ex. Hakulinen et al., 2004, \$ 118). Comme l'impératif, elle exprime l'intention du locuteur qui est orientée vers l'autre. En revanche, elle ne met pas en scène un participant de l'énonciation, mais quelqu'un ou quelque chose qui est extérieur à la relation polaire entre le locuteur et son interlocuteur. Ainsi, l'emploi du jussif n'engage pas le locuteur dans un rapport «face-à-face » (Benveniste, 1966, p. 260).

Dans une perspective guillaumienne, le jussif est un élément virtualisant (Guillaume, 1929): son sémantisme n'inclut pas de structure temporelle qui permettrait de situer l'état de choses par rapport au moment de l'énonciation. De ce fait, il est également sans implication sur la valeur de vérité de l'état de choses : le procès est exprimé à un niveau idéal, non comme un fait ${ }^{3}$. Le jussif se différencie donc par son sémantisme des temps de l'indicatif et du conditionnel, modes construisant un lien temporel entre l'état de choses et le moment de l'énonciation (Peltola, 2011, p. 129-214). L'organisation des modes verbaux finnois peut être illustrée comme un continuum, s'inspirant de l'axe chronogénétique de Guillaume :

\footnotetext{
3 À cet égard, le jussif finnois est comparable aux subjonctifs français et anglais (voir Gosselin, 2005, p. 94-96 ; Leech, 1987 (1971), p. 113-16).
} 
Exemple 2 : Le jussif et les autres modes verbaux du finnois

VIRTUEL

ACTUEL

\begin{tabular}{ccc} 
& & \\
\hline $\begin{array}{c}\text { les formes nominales } \\
\text { du verbe }\end{array}$ & $\begin{array}{c}\text { jussif } \\
\text { impératif } \\
\text { potentiel }\end{array}$ & indicatif \\
conditionnel
\end{tabular}

Le jussif porte une valeur de non-opposition qui peut se situer, sur un plan modal, aussi bien du côté de possible que de celui de nécessaire (Lyons, 1977, p. 832) et qui, de ce fait, donne lieu à toute une série d'interprétations allant de la permission jusqu'à l'ordre, selon le contexte. Nous nous intéressons dans cet article aux usages permissifs de ce mode, c'est-à-dire aux contextes où l'énoncé jussif exprime la nonopposition du locuteur à l'intention d'un tiers ou à une obligation causée par les circonstances. Le locuteur ajuste sa propre volonté par rapport à celles-ci.

Dans l'exemple (1a) présenté ci-dessus, repris sous (3), le contexte précédant les énoncés jussifs permet d'observer que les procès < prendre> et < garder> sont associés à un agent intentionnel, qui est un homme autre que le locuteur A.

Exemple 3 :

(Discours intérieur de A portant sur le départ de sa femme avec un étranger.)

1 (A:) Yhdessä tulivat tähän pihasta. Se mies seisoi tuossa aitaa vasten. [Tous les deux, ils sont venus ici, de la cour ; cet homme se tenait là, contre la clôture.]

2 Joko lienevät sen silloin päättäneet? (...) [L'avaient-ils déjà décidé ((de fuir)) ?(...)]

3 He ovat nuoria ja kauniita. Minä olen vanha ja ruma. [lls sont jeunes et beaux, moi je suis vieux et laid.]

4 Mitäpä hän minusta, kun on semmoinen, joka ottaa. quand être-3 un (comme ça) REL prendre-3 [Pourquoi se soucierait-elle de moi quand c'est un homme comme ça qui la prend.]

Otta-koon! Otta-koon! Pitä-köön!
prendre-Jus $\quad$ prendre-Jus $\quad$ garder-JUS
[Q'il la prenne, qu'il la prenne et qu'il la garde!]

(Aho, 1911, VII)

Avec les énoncés jussifs Ottakoon! Pitäköön! «Qu’il la prenne, qu’il la garde !», (ligne 5), le locuteur exprime qu'il ne s'oppose pas à l'intention de l'autre homme. De plus, faute d'ancrage temporel, les procès s'envisagent comme une possibilité théorique. 
Ce n'est pas leur réalisation qui est pertinente, mais la non-opposition du locuteur à celle-ci.

Il faut noter que les procès auxquels le locuteur s'ajuste figurent, d'après le contexte et nos connaissances du monde, comme a priori désavantageux pour lui. Il est par là même censé s'y opposer.

Grâce à la valeur intentionnelle du jussif, le locuteur se place sur la scène énonciative en tant que participant exerçant un contrôle sur la situation. Il n'est cependant pas l'agent des procès. Ce rôle est assuré par une troisième instance à laquelle le locuteur laisse la responsabilité de la validation ou de la non-validation des procès.

\subsection{Le marqueur discursif $-\mathrm{hAn}$}

Le marqueur discursif - $h A n$ tire sa valeur sémantique du pronom logophorique bän «il/elle LOG 》 qui s'utilise notamment dans le discours rapporté introduit par un verbe du type « dire » (Hagège, 1974; Laitinen, 2002 ; 2005; Roncador, 2006; Boyeldieu, 2013). Un exemple de l'emploi logophorique de bän se trouve en (4a) :

Exemple 4a :

VERBE INTRODUCTEUR PAROLES RAPPORTÉES

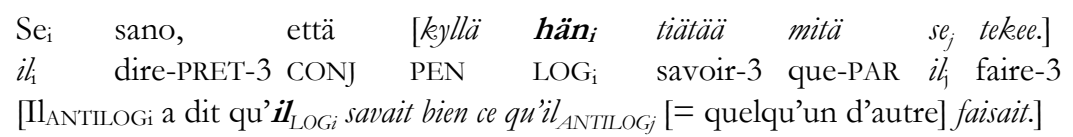

(Setälä, 1883, p. 85)

Exemple 4b :

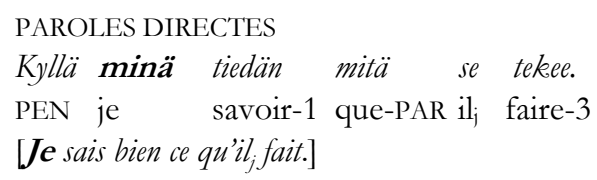

Le logophorique bän « $\mathrm{il}_{\mathrm{LOG}}$ » qui se situe au sein des paroles rapportées pour désigner l'auteur de ces paroles s'interprète comme coréférent avec le sujet du verbe

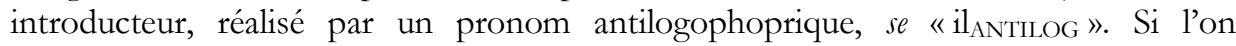
compare le discours rapporté avec le discours direct (ex. 4b), qu'il soit réel ou fictif, on voit que le logophorique bän «il $\mathrm{l}_{\mathrm{LOG}}$ » correspond au pronom de première personne minä « je» des paroles directes. Il réfère alors à un locuteur secondaire. L'emploi du logophorique peut être interprété comme impliquant une prise de distance de la part du locuteur-rapporteur vis-à-vis du contenu du discours rapporté, qui est imputé au locuteur secondaire (Hagège, 1974 ; Stirling, 1994).

À la différence du pronom logophorique bän, qui est une forme autonome, le marqueur discursif - $h A n$ est un enclitique qui s'adapte au vocalisme de sa base (-han $-b \ddot{a} n)$. De plus, $-h A n$ ne réfère pas. Cependant, il a conservé le sens indiciel du pronom bän, c'est-à-dire sa logophoricité : en tant que marqueur discursif, - $h A n$ sert à indiquer que le locuteur n'est pas l'instigateur de l'état de choses qu'il commente (Duvallon, à paraitre). 
Pour éclairer le fonctionnement de $-h A n$ et l'effet de distanciation qu'il crée, considérons l'exemple (5). Il s'agit d'un couple question-réponse où $\mathrm{B}$ refuse d'apporter la réponse attendue à la question de A.

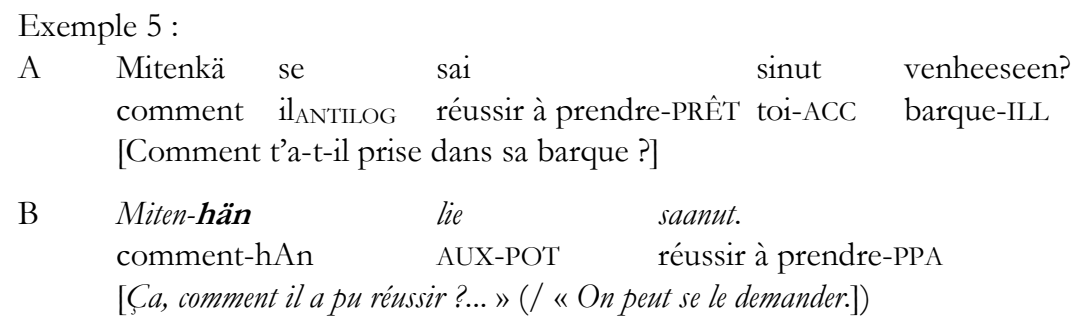

(Aho, 1911, XV)

Le verbe de l'énoncé interrogatif de $\mathrm{A}$ est à l'indicatif et sa forme sujet est réalisée par le pronom antilogophorique se «i $\mathrm{i}_{\mathrm{ANTILOG}}$ ». Autrement dit, le locuteur $\mathrm{A}$ prend en charge la réalité de l'état de choses qu'il énonce («il t’a prise dans sa barque »), l'interrogation partielle (miten «comment») portant sur la façon dont ce procès s'est réalisé.

La réponse de $\mathrm{B}$ reprend le verbe de la question, mais le mode indicatif est remplacé par le potentiel qui exprime, comme le jussif, la modalité théorique (Forsberg, 1998, p. 304 ; voir aussi le schéma (2) ci-dessus) : le procès n’est ni validé ni invalidé, mais évoqué comme une idée. L'énoncé-réponse répète aussi le mot interrogatif miten «comment» auquel est ajouté l'enclitique - $h A n$ qui présente ici, en théorie, une ambiguiité catégorielle: vu l'agencement syntaxique de l'énoncé, on pourrait le considérer aussi comme un pronom sujet qui remplace l'antilogophorique se

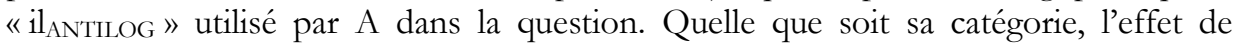
distanciation que $-h A n$ produit est le même. Dans sa réponse, B commente une relation prédicative qu'elle ne prend pas en charge, ce qui est indiqué par - $h A n$; seule la modalité théorique se détermine depuis la position de $\mathrm{B}$ dans la scène énonciative.

Le schéma en (6) illustre les différentes positions de la scène énonciative qui sont en jeu dans les énoncés en $-h A n$ :

Exemple 6 : La scène énonciative

\section{Sx}

ESPACE INTERSUBJECTIF

$$
\left[\begin{array}{lll}
\mathbf{S}_{0} & \mathrm{~S}_{1}
\end{array}\right]
$$

$\mathrm{S}_{0}:$ position du locuteur

$\mathrm{S}_{1}$ : position d'un interlocuteur qui se sépare de la position $\mathrm{S}_{0}$

$S_{X}$ : repère énonciatif impliqué par bän $\sim-h A n$, correspondant à un valideur fictif (« troisième instance ») 
La position du locuteur est notée $\mathbf{S}_{\mathbf{0}}$. Elle fait partie de l'espace intersubjectif où elle se trouve dans un rapport d'altérité avec la position $\mathbf{S}_{1} . \mathbf{S}_{1}$ est la position d'un interlocuteur qui se sépare de la position $\mathbf{S}_{\mathbf{0}}$ pour la remettre en cause (de Vogüé et Paillard, 1987; Paillard 2009). Le marqueur discursif $-h A n$ qui a conservé le sens indiciel du logophorique bän implique un repère énonciatif qui est situé en dehors de l'espace intersubjectif et qui est noté $\mathbf{S}_{\mathbf{X}}$. C'est « une troisième instance » qui échappe à l'altérité des positions $\mathbf{S}_{\mathbf{0}}$ et $\mathbf{S}_{\mathbf{1}}$ (de Vogüé, 1987, p.121-122; de Vogüé et Paillard, 1987, p. 28-31).

Comme on l'a vu dans l'exemple (5), $-\not h A n$ peut s'employer dans un énoncé qui reprend une relation prédicative mise en mots par l'interlocuteur. Grâce à ce marqueur discursif, la responsabilité énonciative quant à la prédication est imputée, non pas à l'interlocuteur, mais à un valideur fictif ${ }^{4}$, non spécifié. Il est à noter que même si l'on pouvait penser que -bän, qui est dans cet exemple une forme potentiellement référentielle, donne une certaine saillance à l'agent du procès comme un valideur «par excellence » de la vérité du procès, il ne s'agit là que d'un effet interprétatif. L'agent du procès n'a pas ici de rôle énonciatif. Le propre de $-b A n$ est donc de mettre en place un repère énonciatif en dehors de l'espace intersubjectif de la scène énonciative ${ }^{5}$. Le rattachement de l'énoncé au repère $\mathbf{S}_{\mathbf{X}}$ sert à indiquer que pour le locuteur, les déterminations de la relation prédicative, notamment sa validation ou non, ne constituent pas un enjeu intersubjectif.

Revenons à l'exemple cité dans l'introduction, repris sous (7), où l'énoncé en $-h A n$ se situe dans le prolongement d'un passage narratif:

Exemple 7 :

(A est une femme abandonnée par son amant, Shemeikka; B est la mère de celui-ci.)

1 B : Shemeikat eivät välitä vanhoista, niillä pitää aina olla nuoria ja aina uusia,

2 niin piti isälläänkin olla.

[Les Shemeikka ne se soucient pas des vieilles, il leur en faut toujours des jeunes et toujours des nouvelles. C'était comme ça pour son père aussi.]

$3 \mathrm{~A}$ : Ja se on teistä, niinkuin olla pitää?

[À votre avis, c'est bien ainsi ?]

4 B : Ei isänsä ensimmäisenä kolmena vuotena kehenkään muuhun koskenut. [Pendant les trois premières années, son père n'a regardé personne d'autre que moi.]

\footnotetext{
4 « [L]e repère fictif, loin de renvoyer à quelque domaine des fictions comme pourrait le suggérer à tort le terme de 'fictif', constitue (...) le point d'où se valide ce qui est extérieur à la problématique du vrai et du faux » (de Vogüé 1987, p. 122).

${ }^{5}$ En effet, même dans le cas des paroles rapportées, le logophorique bän «il $\mathrm{l}_{\mathrm{LOG}}$ » en tant que tel ne construit pas son référent comme doté d'un rôle énonciatif. Sa coréférence avec le sujet du verbe introducteur (ex. 4a), qui n'est en aucun cas obligatoire, est le résultat d'un calcul référentiel (Duvallon, à paraître).
} 
Outi Duvallon et Rea Peltola

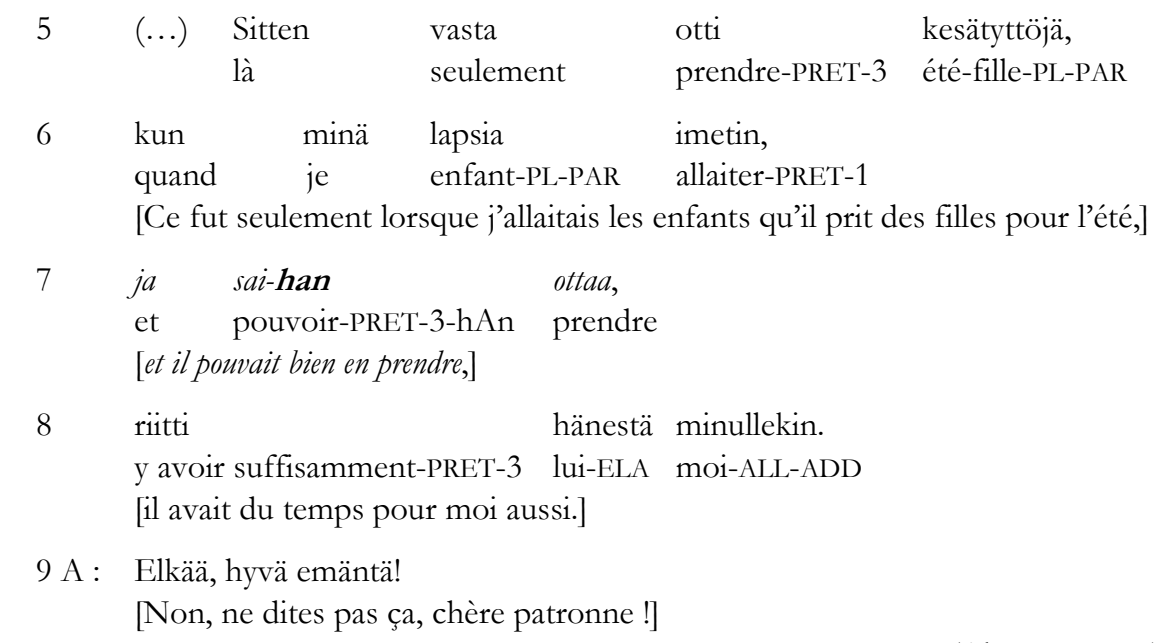

(Aho, 1911, XI)

L'énoncé à la ligne 7, ja saihan ottaa «et il pouvait bien en prendre », reprend le verbe ottaa "prendre» de l'énoncé précédent (ligne 5) en le soumettant à la modalité permissive, exprimée par le verbe modal saada "pouvoir». Cet énoncé permissif suspend la narration pour introduire dans le discours une prise de position qui contraste avec les attentes de l'interlocutrice A (voir lignes 3 et 9) : B accorde son approbation au fait que son mari prenne des filles pour l'été. La fonction de $-b A n$, qui est suffixé au verbe modal, est d'indiquer que $\mathrm{B}$ ne fait que commenter un état de choses $\mathbf{p}$ qui est établi indépendamment d'elle. Comme dans l'exemple (5), la responsabilité énonciative quant à l'existence de $\mathbf{p}$ est déplacée à l'extérieur de l'espace intersubjectif, vers un valideur fictif. Cela évite pour B de se mettre en conflit direct avec son interlocutrice.

\subsection{Partage de la responsabilité énonciative entre deux instances}

Pour récapituler, le marqueur discursif $-h A n$ permet au locuteur de prendre en considération, donc prendre au sérieux (cf. Filippe-Deswelle, 2014), une relation prédicative dont il n'est pas l'instigateur. $-h A n$ indique que la validation première de la prédication est associée à une troisième instance, à un repère énonciatif $\mathbf{S}_{\mathbf{X}}$ situé en dehors de l'espace intersubjectif, le locuteur étant responsable uniquement du mode, quel qu'il soit, sous lequel la prédication est actualisée dans le discours.

Pour ce qui concerne la forme jussive, elle aussi met en place une situation d'énonciation qui implique deux instances, le locuteur et un tiers, c'est-à-dire les positions $\mathbf{S}_{\mathbf{0}}$ et $\mathbf{S}_{\mathbf{X}}$ de la scène énonciative. Cependant, avec la forme jussive, le locuteur prend à son compte la mise en mots du procès, mais le présente comme une possibilité théorique : la responsabilité de sa validation ou non est imputée à un tiers qui peut correspondre à l'argument agent du verbe si le procès est agentif, mais qui, de manière plus générale, est un valideur fictif non spécifié. De ce point de vue, le jussif met en place une situation inverse de celle construite par - $h A n$, comme illustré en (8). 
Exemple 8:

\section{$-h A n$}

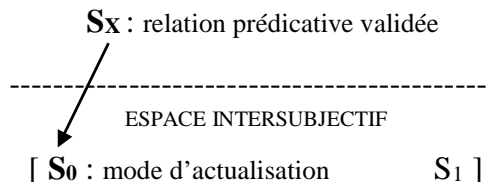

\section{Le jussif}

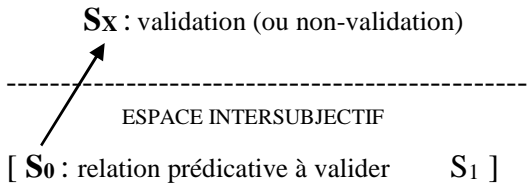

\section{Figures concessives}

Du fait de leur capacité à associer l'énoncé à deux repères énonciatifs, le jussif et le marqueur discursif $-h A n$ apparaissent dans certains contextes similaires, en particulier ceux où émerge une interprétation concessive. Les exemples que nous analyserons ci-dessous se caractérisent par un lien concessif dans deux sens. D'un côté, le locuteur prend en considération un état de choses $\mathbf{p}$ qui n'émane pas de lui et auquel il n'adhère pas complètement (Morel, 1996, p. 15). D'un autre côté, l'énoncé comportant la forme jussive ou $-h A n$, noté énoncé $\boldsymbol{A}$, forme une unité complexe à valeur concessive avec l'énoncé qui le suit, énoncé $\boldsymbol{B}$.

Considérons d'abord l'exemple (9) avec un énoncé jussif :

\section{Exemple 9 :}

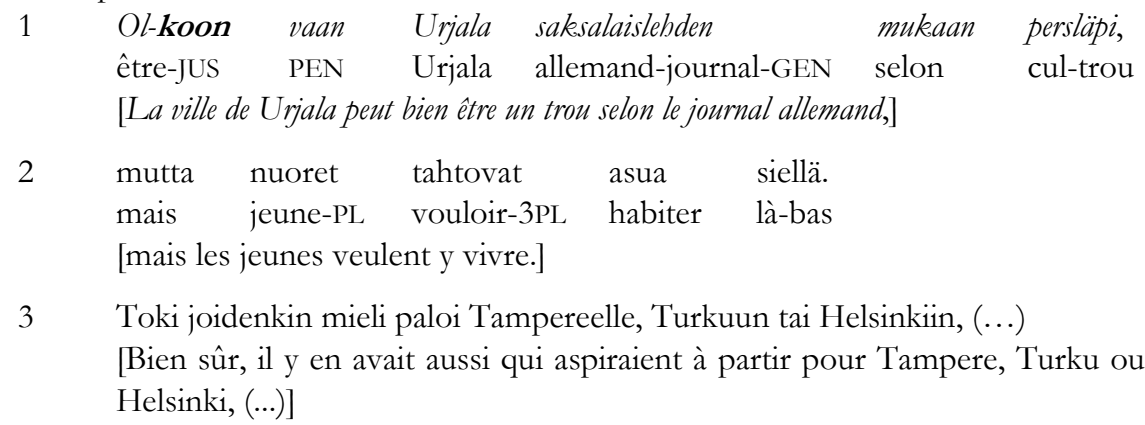

(Skt, Aamu 1999)

La forme jussive est utilisée pour évoquer un état de choses $\mathbf{p}$ déjà établi ( la ville de Urjala est un trou »), dont la source est exprimée par le groupe postpositionnel saksalaislehden mukaan «selon le journal allemand ». Grâce au jussif, la validation de $\mathbf{p}$ est laissée en suspens. Au lieu de déterminer sa valeur de vérité, le locuteur met en avant que sa validité théoriquement possible n’a pas de conséquences sur la validité de l'état de choses $\mathbf{q}$, mis en mots par l'énoncé $\mathbf{B}$, nuoret tabtovat asua siellä «les jeunes veulent y vivre ».

Le lien concessif entre $\mathbf{p}$ et $\mathbf{q}$ se fonde sur la valeur de non-opposition du jussif (Peltola, 2011, p. 185-195). Il s'agit d'une relation qui implique non seulement 
l'inefficacité, mais aussi un conflit (König, 1985, p. 265 ; Ranger, 1998, p. 24-25 ; Culioli, 1999, p. 177-180). Le fait que les deux états de choses puissent, théoriquement, se réaliser simultanément contraste avec ce à quoi on pourrait s'attendre, correspondant à non-q (« les jeunes ne veulent pas habiter dans un trou »). Le rapport de contraste entre $\mathbf{p}$ et $\mathbf{q}$ est marqué par mutta « mais».

L'exemple (10) comporte un énoncé en $-h A n$ :

Exemple 10 :

(Discours intérieur de A portant sur le comportement de sa femme vis-à-vis de lui.)

1 (A :) Ennen se aina toi ruokaa, olipa työpaikka kuinka kaukana tahansa (...)

2 nyt katsoo vanhaksi: "Sen kanttura, sen vääräsääri... sen väkäleuka". [Autrefois, aussi loin qu'était le lieu de mon travail, elle [= la femme de A] m'apportait toujours mon repas (...) maintenant elle me prend pour un vieux : 'Espèce de vieux mal fichu, sale boiteux... sale menton en galoche !']

3

$\begin{array}{ll}\text { Saattaa-han } & \text { olla, } \\ \text { pouvoir-3-hAn } & \text { etre }\end{array}$

[C'est bien possible ['(cela) peut-h $A$ h être'],]

4 mutta (...) onko se nyt siinä, minkä näköinen mies mais être-Q ce PEN DEM-INE quoi-GEN ayant l'air homme

5 on, tai minkä ikäinen, kunhan vaan on mies? être-3 ou combien-GEN âgé si seulement PEN être-3 homme [mais (...) qu'importe l'aspect physique ou l'âge de l'homme, si seulement c'est un homme ?]

6 Eikö se ole siinä, mihin kykenee

NEG-3-Q ce être DEM-INE quoi-ILL être capable-3

7 ja mitä saa aikaan?

et que-PAR produire-3

[Sa valeur ne se mesure-t-elle pas à ce dont il est capable et à ce qu'il fait ?]

(Aho, 1911, I)

L'énoncé en - $h A n$ (ligne 3) qui constitue le début de la réaction de $\mathrm{A}$ à des moqueries de sa femme qu'il cite dans son discours intérieur est formé du modal épistémique saattaa "pouvoir», porteur de $-h A n$, et du verbe support olla "être» (saattaaban olla "c'est bien possible»). Le modal saattaa "pouvoir» exprime un jugement de la part du locuteur sur la valeur de vérité des prédications citées. La particule $-h A n$ signifie que le locuteur ne prend pas en charge ces prédications, mais seulement la modalité qu'il projette sur elles. Le locuteur reconnait donc comme possibles les idées émanant d'un autre locuteur. L'énoncé en - $-h A n$ est suivi du coordonnant mutta « mais » qui introduit des interrogations (lignes 4-7) qui produisent un effet de rectification : il s'agit de mettre en doute les conclusions que l'on pourrait tirer de l'énoncé concédé.

Ce type d'enchaînement discursif produit le schéma d'une concession argumentative (Morel, 1996, p. 15-18; Couper-Kuhlen et Thompson, 2000) : le fait de 
prendre en considération le point de vue d'autrui sert, pour le locuteur, de moyen d'introduire son propre point de vue dans le discours. Le mode jussif et le marqueur discursif - $h A n$ sont deux formes concurrentes permettant au locuteur d'éviter une remise en cause directe d'un état de choses qui n'est pas conforme au point de vue qu'il souhaite mettre en avant.

\section{Figures causales}

Si la forme jussive et - $h A n$ se combinent difficilement à l'intérieur d'un énoncé, ils peuvent cependant se suivre dans le texte de sorte qu'un lien de causalité s'établit entre eux. Le rapport causal peut unir la cause et la conséquence, dans un sens étroit, mais l'un des éléments de cette relation sémantique peut tout aussi bien correspondre à une condition, à un résultat, à un but ou à une raison (voir par ex. Rudolph, 1996, p. 24-26 ; Cristofaro, 2011). Dans les figures formées de l'énoncé jussif et de l'énoncé en - $h A n$, le rapport causal ne concerne pas que les états de choses en tant que tels; il implique aussi les repères énonciatifs, la position du locuteur et celle d'un tiers.

Dans l'exemple (11), le lien causal s'opère au niveau discursif (cf. par ex. Debaisieux, 2006 ; Couper-Kuhlen, 1996). Le contenu du deuxième élément de la relation causale est envisagé comme une raison. Il fournit une justification à l'attitude du locuteur exprimée dans l'énoncé précédent.

Exemple 11 :

1 Marja istuu eräänä sateisena päivänä (...) tuvassa (...) kutoen pientä

2 lapsen sukkaa.

[Un jour de pluie (...) Marja [ $[=\mathrm{A}]$ est assise dans la salle $(\ldots)$, elle tricote un chausson de bébé.]

3 (A:) Kysy-köön, jos kysyy, sanon: kudon lapselleni. demander-Jus si demander-3 dire-1 tricoter-1 enfant-ALL-POS.1 [Qu'il m'interroge, s'il le veut. Je lui dirai : Je tricote pour mon petit.]

4

En jaksa sitä salata... enkä välitä...

NEG-1 avoir la force le-PAR cacher NEG-1-COOR se préoccuper

[Je ne peux pas le cacher plus longtemps... je m'en fiche...]

5 saa-koon tietää.. saa-han se sen kuitenkin.

INCH-JUS savoir INCH-3-hAn il le-GEN malgré moi

[qu'il le sache... il finira bien par le savoir.]

(Aho, 1911, XV)

Les énoncés aux lignes 3-5 constituent un discours intérieur de A qui porte sur une éventuelle interrogation de la part de son mari. La forme jussive kysyköön «qu'il m'interroge » (ligne 3) exprime la non-opposition de A à l'état de choses p qui dépend de l'intention de son interlocuteur potentiel. Cette non-opposition ainsi que le contenu de la réponse envisagée contrastent avec la volonté de A de cacher l'existence de l'enfant qu'elle a eu avec un autre homme. 
Le deuxième énoncé jussif à ligne 5 saakoon tietää « qu’il le sache » évoque, à titre de possibilité théorique, un état de choses q qui serait la conséquence de l'échange hypothétique. Étant donné que cet état de choses q contraste avec l'intention initiale de la locutrice, le fait de ne pas s'y opposer implique une attitude d'indifférence, qui est lexicalisée par l'énoncé en välitä « je m'en fiche » précédant l'énoncé jussif.

L'énoncé au jussif est suivi d'un énoncé en - $h A n$ (ligne 5) qui reprend le même verbe à l'indicatif. Le marqueur discursif $-h A n$ signifie que la validation première de l'état de choses $\mathbf{q}$ 《il finira par le savoir» est associée cette fois au repère $\mathbf{S}_{\mathbf{X}}$ : $\mathbf{q}$ est établi indépendamment de la position de A. La modalité affirmative et le mode indicatif du verbe témoignent de l'accord de A avec cet état de choses; la seule trace de son intention initiale se trouve dans l'expression concessive kuitenkin, glosable par «malgré moi».

Cet exemple illustre un enchaînement discursif où le locuteur abandonne son intention afin de se conformer à une situation qui s'impose à lui. Le changement d'orientation se fait au moyen de la forme jussive avec laquelle le locuteur met en mots un état de choses qui ne lui est pas avantageux, et il est entériné par l'énoncé en - $h A n$ qui reformule la situation comme étant inévitable et indiscutable. Ainsi, l'énoncé en $-h A n$ se trouve dans un rapport de justification avec l'énoncé jussif.

Dans le deuxième exemple de figures causales (exemple (12)), le lien entre l'énoncé jussif et l'énoncé en - $h A n$ est conditionnel. Les deux énoncés qui sont unis par une conjonction forment une construction complexe.

Exemple 12:

1 Vielä suuremmalla varmuudella odotti Juha nyt Marjaa.(...)

[Avec une certitude plus grande encore, Juha attendait maintenant Marja.

$(\ldots)]$

2 Se tulee, tuli-pa-han, milloin tulee, elle revenir-3 revenir-PRET-3-PEN-hAn quand revenir-3 [Elle reviendra quand elle voudra,]

3 mutta se tulee.

mais elle revenir-3

[mais elle reviendra.]

4 Ja tul-koon-pa vaikea keymmenen vuoden kuluttua,

et revenir-JUS-PEN FOC dix-GEN an-GEN au bout

[Et elle peut bien revenir dans dix ans,]

5 kunhan tulee.

CONJ-hAn revenir-3

[pourvu qu'elle revienne.]

(Aho, 1911, XIII)

L'énoncé jussif (ligne 4) est précédé d'énoncés par lesquels le locuteur exprime, dans son discours intérieur, sa conviction sur le retour de sa femme, partie sans explication. Il s'agit de désigner un procès futur validé (ligne 2 : se tulee "elle reviendra »), mais avec une indétermination concernant son ancrage temporel. Que le 
choix de la détermination temporelle du retour soit laissé à l'agent du procès ou à une autre instance quelconque est indiqué par l'énoncé incident à valeur concessive qui comporte - $h A n$, tulipahan milloin tulee "(peu importe) quand elle voudra (revenir)» (ligne 2). Enfin, l'énoncé jussif à la ligne 4 reprend le verbe tulla « revenir» pour présenter le même procès à un état virtuel, associé un repère temporel (kymmenen vuoden kuluttua «dans dix ans») qui est sous la portée d'une particule focalisante, vaikka, et qui s'interprète comme une borne extrême (Duvallon et Peltola, 2014). Cet énoncé, tout comme l'énoncé incident en - $b A n$, traduit l'ajustement du locuteur à l'intention de sa femme.

Cependant, l'ajustement est soumis à une condition. L'énoncé jussif est suivi d'un énoncé introduit par la conjonction kunhan "pourvu que» (ligne 5) qui est composée de la conjonction conditionnelle et causale kun (Herlin, 1996) et de l'élément $-h A n$. L'énoncé en kunhan pose une condition nécessaire et suffisante à la non-opposition du locuteur à une attente pouvant durer jusqu’à dix ans. La présence de $-b A n$ signifie qu'il ne s'agit plus d'exprimer la conviction du locuteur, mais d'actualiser dans le discours un état de choses relevant de la responsabilité d'une troisième instance $\mathbf{S}_{\mathbf{x}}$, non spécifiée, mais que l'on peut interprétée ici comme correspondant potentiellement à l'agent du procès, la femme attendue. Pour résumer, la non-opposition du locuteur à une possibilité qui lui est peu avantageuse est conditionnée par la réalisation d'un état de choses qui n'est pas sous son contrôle.

\section{Conclusion}

Nous avons étudié, dans cet article, le mode jussif et le marqueur discursif - $h A n$ en tant que moyens linguistiques permettant au locuteur de se distancier d'un sens préconstruit dans le discours. Tout en soulignant la différence sémantique fondamentale entre ces formes, nous avons tenté de montrer que, dans les deux cas, l'effet de distanciation se base sur la mise en place d'un double repérage, la responsabilité énonciative étant partagée entre le locuteur et un tiers.

Ce procédé de distanciation rend ces formes propices à l'expression de la concession, qui est une relation mettant en rapport tant les énonciateurs que les états de choses et leur réalisation (Ranger, 1998, p. 3). Dans les figures concessives, où le jussif et $-h A n$ sont des formes concurrentes, le locuteur prend en considération le point de vue d'un tiers qui lui est a priori peu avantageux pour réorienter ensuite le discours vers son propre point de vue.

En tant que formes co-présentes, le jussif et le marqueur discursif $-h A n$ conviennent à l'établissement de liens de type causal. Comme dans le cas de la concession, il s'agit là de relations impliquant la dissociation entre la position du locuteur et celle d'une troisième instance qui est un valideur fictif non spécifié, mais auquel l'interprétation peut faire correspondre, dans certains cas, l'agent du procès. Les figures causales permettent au locuteur de se conformer à un état de choses qui s'impose à lui et qui est en conflit avec son intention première.

De par le double rattachement énonciatif, le jussif et - $h A n$ créent, chacun à sa manière, un réseau complexe d'intentions : l'énoncé est placé au croisement des sens attendu et inattendu. Avec le jussif, le locuteur se place sur la scène énonciative 
comme un participant ayant un certain contrôle sur la situation, puisqu'il a le choix de s'opposer ou de s'ajuster aux intentions associées à la troisième instance, alors qu'avec -hAn, son rôle est réduit à celui qui actualise dans le discours des intentions premièrement validées indépendamment de lui.

\begin{abstract}
Abréviations
ACC accusatif ; ADD particule additive ; ALL allatif ; ANTILOG antilogophorique ; AUX auxiliaire du passé composé; CONJ conjonction; COOR coordonnant; DEM démonstratif; FOC particule focalisante; GEN génitif; ILL illatif; INCH auxiliaire inchoatif ; INE inessif ; JUS jussif ; LOG logophorique ; NEG verbe négatif ; PAR partitif ; PEN particule énonciative; PL pluriel ; POS suffixe possessif; POT potentiel ; PPA participe passé; PRET prétérit; $Q$ suffixe interrogatif; REL relatif; 1 première personne ; 2 deuxième personne ; 3 troisième personne.
\end{abstract}

\title{
Sources des exemples cités
}

Aho, J., 1911, Juha, Helsinki, Kustannusyhtiö Otavan kirjapaino.

Setälä, E. N., 1883, Lauseopillinen tutkimus Koillis-Satakunnan kansankielestä, Helsinki, Société de littérature finnoise.

Skt = Suomen kielen tekstikokoelma - "Corpus électronique de textes en finnois », établi par l'Institut de recherche pour les langues de Finlande, le département de linguistique générale de l'Université de Helsinki, le département des langues étrangères de l'Université de Joensuu et CSC - Scientific Computing Ltd, disponible par le site internet du CSC, <http://www.csc.fi>. 


\section{Bibliographie}

Benveniste, É., 1966, Problèmes de linguistique générale 1, Paris, Gallimard.

BOYELDIEU, P., 2013, "Introduction », in Boyeldieu, P. (éd.), Logophorique et discours rapporté en Afrique centrale, Louvain-Paris, Peeters, p. 9-36.

COUPER-KuHLEN, E., 1996, "Intonation and clause combining in discourse: the case of because », Pragmatics, n 6, p. 389-426.

Couper-Kuhlen, E. and Thompson S. A., 2000, "Concessive patterns in conversation », in Couper-Kuhlen, E. et Kortmann, B. (eds), Cause, Condition, Concession, Contrast: Cognitive and Discourse Perspectives, Berlin, Mouton de Gruyter, p. 381-410.

Cristofaro, S., 2011, «Reason Clauses », in Dryer, M. S. and Haspelmath, M. (eds), The World Atlas of Language Structures Online, chapitre 127, Munich, Max Planck Digital Library, disponible en ligne : http://wals.info/chapter/127 (le 17 juin 2013).

Culioli, A., 1999, Pour une linguistique de l'énonciation, tome 3, Paris, Ophrys.

DE VOGÜÉ, S., 1987, «La conjonction si et la question de l'homonymie », BULAG 13, p. 105189.

DE VogÜÉ, S. et PAILlard D., 1987, « Modes de présence de l'autre », in Les particules énonciatives en russe contemporaine 2, Paris, Institut d'Etudes Slaves, p. 11-37.

Debaisieux, J.-M., 2006, «La distinction entre dépendance grammaticale et dépendance macrosyntaxique comme moyen de résoudre les paradoxes de la subordination », Faits de langues, $\mathrm{n}^{\circ} 28$, p. 119-132.

Dendale, P. et Coltier, D., 2011, « Présentation », in Dendale, P. et Coltier, D. (éds), La prise en charge énonciative :études théoriques et empiriques, Bruxelles, De Boeck / Duculot, p. 7-17.

DUVALLON, O., à paraitre, «Le marqueur discursif finnois - $h A n$ : double repérage énonciatif », Faits de langues, $\mathrm{n}^{\circ} 43$, p. 87-114.

Duvallon, O. et Peltola, R., 2014, «Les énoncés concessifs en finnois : hypothèse sur le passage du virtuel au réel », Travaux linguistiques du CerLico, n 25, p. 207-226.

FilipPi-Deswelle, C., 2014, « Du réel à l'irréel : even if et la construction de tous les possibles », Travaux linguistiques du CerLico, n 25, p. 191-206.

FORSBERG, H., 1998, Suomen murteiden potentiaali: muoto ja merkitys, Helsinki, Société de littérature finnoise.

FORSBERG, H., à paraitre, «Imperatiivi kannanottona: varoituksesta paheksuntaan », in Haakana, M. et Sorjonen, M.-L. (eds), Tunteet käytössä: tutkimuksia kielenkäytön affektiivisundesta, Helsinki, Société de littérature finnoise.

Forsman Svensson, P., 2003, «Tuleppas siihen nenäilemään: Pragmaattiset liitepartikkelit 1600- ja 1700-luvun diskurssissa », in Forsman Svensson, P. (ed.), Suomen kielen syntaksin kebitystä 1600-luvulta 1800-luvun alkupuolelle, Helsinki, Helsingin yliopiston kielikeskus, p. $52-82$.

Gosselin, L., 2005, Temporalité et modalité, Bruxelles, Duculot. 
Guillaume, G., 1929, Temps et verbe: théorie des aspects, des modes et des temps, Paris, Édouard Champion.

HagÈge, C., 1974, «Les pronoms logophoriques », Bulletin de la Société de Linguistique de Paris, LXIX/1, p. 287-310.

Hakulinen, A., Vilkuna, M., Korhonen, R., Koivisto, V., Heinonen, T. R. et Alho, I., 2004, Iso suomen kielioppi, Helsinki, Société de littérature finnoise, disponible en ligne: http:/ / scripta.kotus.fi/visk/etusivu.php (le 17 juin 2013).

HAKULINEN, L. 1999 (1951), Luennot suomen kielen partikkeleista, Lauranto Y. et Lehtinen T. (eds), Helsinki, Helsingin yliopiston suomen kielen laitos.

HerLiN, I., 1996, Suomen kun, Helsinki, Société de littérature finnoise.

KÖNIG, E., 1985, «Where do concessives come from? On the development of concessive connectives ", in Fisiak, J. (ed.), Historical Semantics, Historical Word-Formation, Berlin /New York /Amsterdam, Mouton, p. 263-282.

LAITINEN, L., 2002, «From logophoric pronoun to discourse particle: a case study of Finnish and Saami », in Wischer, I. et Diewald, G. (eds), New Reflections on Grammaticalization, Amsterdam, Benjamins, p. 327-344.

LAitinen, L., 2005. «Hän, the third speech act pronoun in Finnish », in Laury, R. (ed.), Minimal Reference: the Use of Pronouns in Finnish and Estonien Discourse, Helsinki, Sociéte de littérature finnoise, p. 75-106.

LEECH, G. N., 1987 (1971), Meaning and the English Verb, London/New York, Longman.

LYONS, J., 1977, Semantics 2, Cambridge, Cambridge University Press.

MOREL, M.-A., 1996, La concession en français, Paris, Ophrys.

PAillard, D., 2009, «Prise en charge, commitment ou scène énonciative », Langue française, 162, p. 109-128.

Peltola, R., 2011, Cohésion modale et subordination: le conditionnel et le jussif finnois au miroir de la valeur sémantique et discursive du subjonctif français, thèse de doctorat, Helsinki, Département de finnois et des langues et littératures finno-ougriennes et nordiques de l'Université de Helsinki, disponible en ligne : http://urn.fi/URN:ISBN: 978-952-10-7376-2/ (le 17 juin 2013).

RANGER, G., 1998, "Les constructions concessives en anglais : une approche énonciative », Cabiers de recherche, Numéro spécial, Paris, Ophrys.

RONCADOR, M., von, 2006, «Logophoric pronouns », in Brown, K. (ed.), Encyclopedia of Language and Linguistics, Second edition, vol. 7, Amsterdam, Elsevier, p. 312-315.

Rudolph, E., 1996, Contrast: Adversative and Concessive Relations and Their Expressions in English, German, Spanish, Portuguese on Sentence and Text Level, Berlin/New York, Walter de Gruyter.

SETÄLÄ, E. N., 1883, Lauseopillinen tutkimus Koillis-Satakunnan kansankielestä, Helsinki, Société de littérature finnoise.

STIRLING, L., 1994, "Logophoricity and long-distance reflexives ", in Asher, R. E. (ed.), The Encyclopedia of Language and Linguistics, vol. 4, Oxford, Pergamon, p. 2302-2306. 